\title{
LOS MAMÍFEROS FÓSILES DEL DISTRITO DE PUENTE DE PIEDRA (XENARTHRA, GLYPTODONTIDAE; ARTIODACTYLA, CAMELIDAE, LAMINI), GRECIA, PROVINCIA DE ALAJUELA, COSTA RICA
}

\author{
FOSSIL MAMMALS FROM PUENTE DE PIEDRA DISTRICT (XENARTHRA, \\ GLYPTODONTIDAE; ARTIODACTYLA, CAMELIDAE, LAMINI), GRECIA, \\ ALAJUELA PROVINCE, COSTA RICA
}

\author{
Eduardo A. Pérez \\ Dirección de Geología, Ministerio de Ambiente y Energía. \\ Apartado Postal 62-1009, San José Costa Rica \\ eperez@costarricense.cr
}

(Recibido: 27/03/2013 ; aceptado: 26/11/2013)

\begin{abstract}
It is described the second fossil record of the family Camelidae and the first of the genus Palaeolama for Costa Rica, which was found together with Glyptotherium plates. This association provides new data about the Great American Biotic Interchange. The material fossil assigned to Camelidae consists of a rear left metapodial. Based on previous studies of fossils from nearby areas these new records are assigned an Early Irvigtonian age.

Keywords: Xenarthra, Glyptodontidae, Camelidae, Lamini, Late Cenozoic, Alajuela, Costa Rica.

RESUMEN: Se presenta el segundo registro fósil de la familia Camelidae, y primero del género Palaeolama para Costa Rica, hallado junto a placas de Glyptotherium. Esta asociación proporciona nuevos datos del Gran Intercambio Biótico Americano. El fósil del Camelidae consiste de un metapodial posterior izquierdo. Con base en estudios anteriores de hallazgos de mamíferos fósiles en localidades cercanas se le asigna una edad Irvingtoniano Temprano.

Palabras clave: Xenarthra, Glyptodontidae, Camelidae, Lamini, Cenozoico Tardío, Alajuela, Costa Rica.
\end{abstract}




\section{INTRODUCCIÓN}

El sitio del hallazgo se ubica en el Tajo la Pista, localizado en el distrito 07 de Puente de Piedra de Grecia en la provincia de Alajuela, aproximadamente $500 \mathrm{~m}$ al sur del puente del Río Colorado sobre la autopista Bernardo Soto, entre las coordenadas Lambert Norte 222000-222500 Norte y las 496500-497000 Este, de la Hoja "Naranjo" del Instituto Geográfico Nacional, escala 1:50 000 (Fig. 1). El material de estudio fue recuperado en sedimentos asociados al paleolacustre de Palmares por el Dr. Buriánek. Dichos restos fueron trasladados a la Dirección de Geología y Minas del MINAE.

Asociado a los depósitos sedimentarios del Lacustre de Palmares se han descrito varios restos de megamamíferos plio-pleistocénicos entre los que destacan Mixotoxodon larensis, Eremotherium laurillardi, Pachyarmatherium leiseyi, Glyptotherium arizonae y restos aún no descritos de gomfoterios y tortuga (Laurito, 1993. Laurito et al., 2005, Valerio \& Laurito, 2004, 2011a).

\section{PALEONTOLOGÍA ARTIODACTYLA}

Orden Artiodactyla Owen, 1848

Suborden Tylopoda Illiger, 1811

Familia Camelidae Gray, 1821

Subfamilia Camelinae Gray, 1821

Tribu Lamini Webb, 1965

Palaeolama Gervais, 1867

\section{Palaeolama mirifica (Simpson, 1929)}

Sinonimia

- Tanupolama mirifica nueva especie en Simpson, 1929, pág. 593-596.

- Tanupolama mirifica Simpson, 1929 en Hibbard \& Dalquest, 1962, págs. 89-91.

- Tanupolama mirifica Simpson, 1929 en Slaughter, 1966, págs. 83-86.

- Paleolama mirifica (Simpson, 1929) en Webb, 1974b, pág. 182-196, fig. 9.8C.

- Paleolama cf. P. mirifica (Simpson, 1929) en Roth \& Laerm, 1980, pág. 19.

- Paleolama mirifica (Simpson, 1929) en Webb \& Stehli, 1995, pág. 625-631, fig. 4D.

- Paleolama mirifica (Simpson, 1929) en Hulbert \& Pratt, 1998, pág. 423.

- Paleolama mirifica (Simpson, 1929) en Webb, 2001a, pág. 264, fig. 13.29C y pág. 266 fig. 13.31F.

\section{Material referido}

El material se encuentra depositado en la colección de fósiles de la Escuela Centroamericana de Geología de la Universidad de Costa Rica y corresponde a un metapodial trasero izquierdo constituido por él: tarso izquierdo, fig. 2A que comprende el CF-5772 un maléolo; el CF-5773 un astrágalo; el CF-5774 un cuboide; el CF-5775 un navicular y el CF-5776 un ectomesocuneiforme; además del CF-5777 un metatarso; el CF-5778 una III falange proximal del pie y el CF-5779 una III falange media del pie.

\section{Descripción del material}

Maléolo lateral - CF-5772: De forma rectangular, comprimido lateralmente, se articula con la tibia, el astrágalo y calcáneo, con un largo de $38,30 \mathrm{~mm}$. La superficie proximal presenta una pequeña espina fragmentada que se articula con la tibia y en la parte distal presenta hacia el centro una notable concavidad (ver figs. 2B y C).

Astrágalo - CF-5773: posee forma rectangular y esbelta, con la tróclea proximal más alta y estrecha que la tróclea distal, el ancho de la tróclea proximal es de 40,22 mm y está compuesta de dos crestas semicirculares que se articulan con la tibia y el peroné; la cresta o cóndilo lateral es más alta que la medial y están separadas por un profundo y ancho valle tibial (ver fig. 2D).

El ancho de la tróclea distal es de $46,35 \mathrm{~mm}$ y está compuesta de dos cóndilos divididos por un surco, esta faceta se articula con el navicular y el cuboide (fig. 2A), el cóndilo navicular es un poco más largo que el cóndilo cuboidal. En la cara anterior del astrágalo, se presenta una fosa inte- 


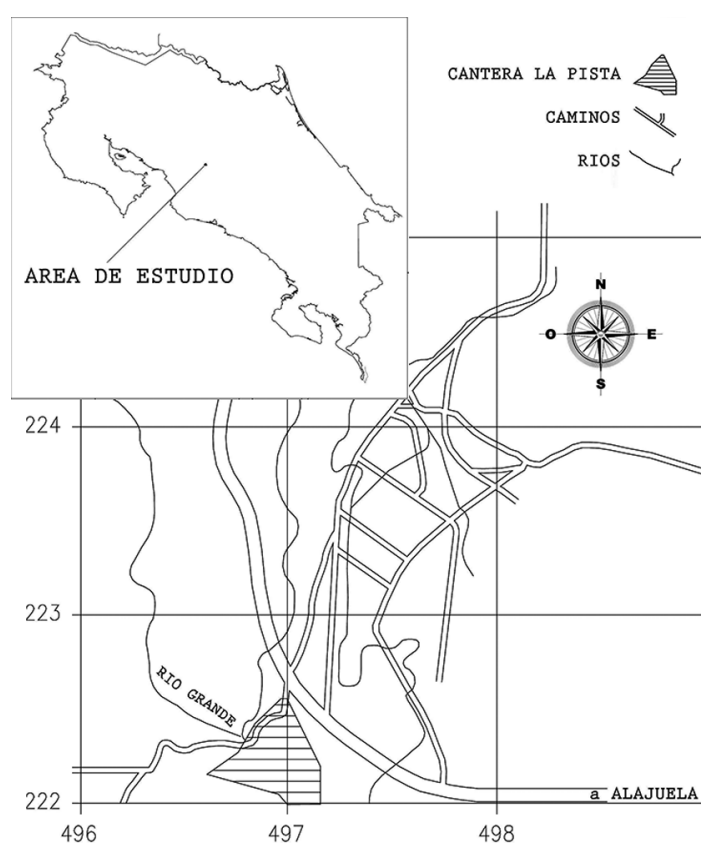

Fig. 1: Ubicación del Tajo La Pista, Grecia (mapa base: Hoja Naranjo, IGN, escala 1:50 000); digitalizó: C. Ramírez (DGM).

rarticular de forma ovoide, amplia y profunda que separa las trócleas.

La cara plantar del astrágalo (fig. 2E) presenta un área protuberante convexa, llamada faceta sustentacular, en la parte distal de esta faceta se ubica una hendidura profunda, denominada fosa sustentacular.

El lado lateral es sigmoide (fig. 2F) y mide $67,50 \mathrm{~mm}$ de largo siendo más alto que el lado medial, más rugoso y excavado.

El lado medial, más corto y ovalado (fig. $2 \mathrm{G})$, mide $62,40 \mathrm{~mm}$ de altura, la parte más ancha se ubica en la zona proximal con $34,93 \mathrm{~mm}$ de ancho, y se extiende sobre la faceta sustentacular.

Cuboide - CF-5774: Hueso pequeño ligeramente trapezoidal y alargado, en buen estado de preservación, con un largo de 47,00 mm, la parte anterior mide $33,20 \mathrm{~mm}$ y es más ancha que la plantar que mide $28,70 \mathrm{~mm}$.

En la superficie proximal se ubica una concavidad que se articula con el cóndilo lateral distal del astrágalo (fig. 2H), en tanto que en la superficie distal (fig. 2I) se observan las facetas que se articulan con el metatarso IV; y en el lado medial se observan las articulaciones que limitan con el navicular y el ectomesocuneiforme.

Navicular - CF-5775: Hueso corto ovalado con un largo de 41,50 $\mathrm{mm}$ con la cara proximal cóncava (fig. 2J) y se articula con el cóndilo medial distal del astrágalo, la cara distal (fig. 2K) es semiplana y se ubica sobre el ectomesocuneiforme. El lado lateral se acomoda con la zona superior medial del cuboide.

Ectomesocuneiforme - CF-5776: Hueso pequeño semicircular, su cara proximal (fig. 2L) es un poco más larga que la distal, aproximadamente $30,00 \mathrm{~mm}$. La cara proximal se une con el navicular y la distal (fig. 2M) se une con la faceta proximal del metatarso (dedo III), su lado lateral es colindante con la parte medial distal del cuboide.

Metatarso fragmentado con los cóndilos III y IV - CF-5777: metatarso incompleto de $213 \mathrm{~mm}$ de largo sin incluir las medidas de los cóndilos (figs. 3A y D), en el que falta la faceta proximal, ésta última se articula con el ectomesocuneiforme y el cuboide; el metatarso es corto y robusto, por la suma del ancho del ectomesocuneiforme y del cuboide se infiere un ancho proximal del metatarso de 53,00 mm. Los cóndilos distales están presentes pero fragmentados y separados del hueso principal, son divergentes y robustos, se infiere para ellos un ancho aproximado de $63,00 \mathrm{~mm}$. El cóndilo III tiene un ancho de $30,00 \mathrm{~mm}$ y el IV $29,00 \mathrm{~mm}$ (figs. 3B, C, E, F).

III Falange proximal del pie - CF-5778: hueso corto y robusto, fragmentado con un largo de $83,00 \mathrm{~mm}$, la faceta articular proximal mide $31,00 \mathrm{~mm}$ de ancho y la tróclea distal con una sola carilla fragmentada, se le infiere una anchura distal de $25 \mathrm{~mm}$ aproximadamente, esta es la que se articula con la falange media (figs. 3G-I).

III Falange media del pie - CF-5779: hueso completo, pequeño y robusto con un largo de 48,29 mm, en la zona proximal se observa la cresta sagital que la divide en 2 cavidades, su ancho es de $26,70 \mathrm{~mm}$, la parte distal presenta una sola carilla, la cual es más pequeña que la proximal con un ancho de 17,00 mm (figs. 3J - L). 


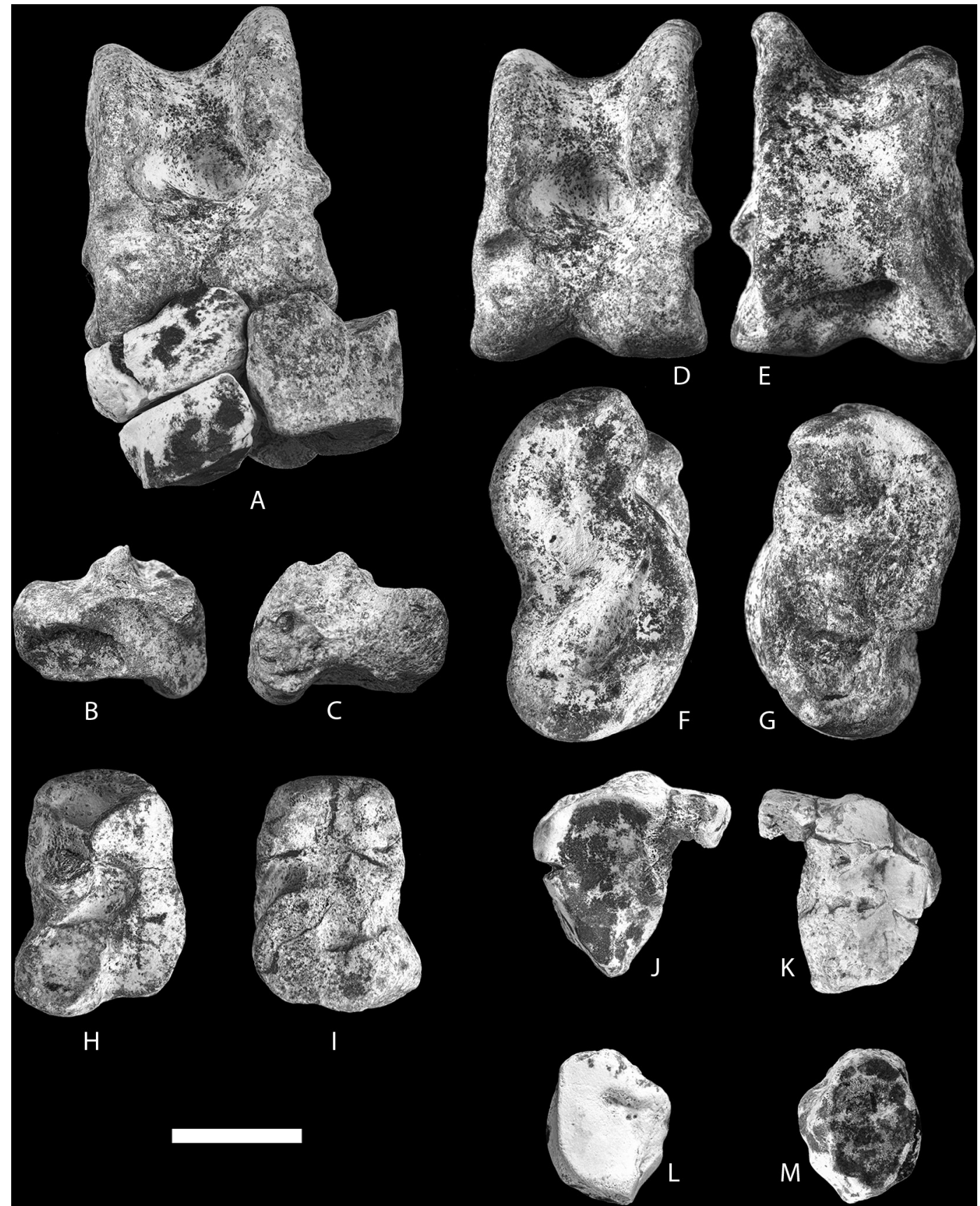

Fi. 2: Palaeolama mirifica (Simpson, 1929): A. vista anterior del tarso izquierdo; CF-5772 = maléolo en vistas: B. lateral y C. medial; CF-5773 = astrágalo en vistas: D. anterior, E. plantar, F. lateral, G. medial; CF-5774 = cuboide en vistas: H. proximal e I. distal; CF-5775 = navicular en vistas: J. proximal y K. distal; CF-5776 = ectomesocuneiforme en vistas: L. proximal y M. distal. Escala visual $3 \mathrm{~cm}$. 


\section{Discusión}

Los camélidos se originaron en Norte América, y son indicativos del Gran Intercambio Americano que se inicia con el cierre del Istmo de Panamá (Webb \& Stehli, 1995).

Anterior al presente hallazgo se registran dos camélidos del género Hemiuchenia en Costa Rica, asociados a los fósiles de Caballos presentes en la Formación Curré, del cantón de Coto Brus, provincia de Puntarenas (Laurito \& Valerio 2010; 2012).

El primer hallazgo de Palaeolama sp. en América Central fue identificado en la localidad de vertebrados fósiles del Río Tomayate de El Salvador (Cisneros, 2005).

Fundamentado en las características de un metatarsiano de proporciones relativamente robustas y los rasgos y dimensiones del astrágalo recuperado (cf. los criterios dados por Webb, 1974b) se identifica al ejemplar del presente estudio como perteneciente a la especies Norteamericana Palaeolama mirifica (Simpson, 1929).

\section{Distribución paleogeográfica}

En relación con el género Palaeolama, solo se acepta la existencia en América del Norte de la especie Palaeolama mirifica o su sinónimo Tanupolama mirifica, presentes en el Pleistoceno de Seminole Field, Pinellas County de la Florida (Simpson, 1929) que según Webb (1974b) corresponde al Rancholabreano Tardío; Pleistoceno Temprano (Irvingtoniano Temprano) de la localidad de Leisey Shell Pits, Hillsboroungh County (Webb \& Stehli, 1995) y el hallazgo con datación más antigua en La Florida que se localiza en Inglis 1C, Citrus County del Irvingtoniano Temprano (Ruez, 2001, 2002, 2005). Para el Pleistoceno de Texas, se registra en la localidad de Seymour Formation en Knox County (Hibbard \& Dalquest, 1962) y en Moore Pit Local Fauna (Slaughter, 1966); en el Rancholabreano de Carolina del Sur, Edisto Island (Roth \& Laern, 1980); en el Rancholabreano de Mayfair Site, Chatham County, Georgia (Hulbert \& Pratt, 1998) y en América Central se registra a nivel de género en el Irvingtoniano del Municipio de Apopa en El Salvador (Cisneros, 2005).

\section{PALEONTOLOGÍA XENARTHRA}

Orden Xenarthra Cope, 1889

Suborden Cingulata Illiger 1811

Superfamilia Glyptodontoidea Gray, 1869

Familia Glyptodontidae Gray, 1869

Subfamilia Glyptodontinae Gray, 1869

Tribu Glyptotheriini Castellanos, 1953

Género Glyptotherium Osborn, 1903

Glyptotherium aff. G. arizonae Gidley, 1926

Sinonimia:

- Glyptotherium sp. cf. G. arizonae Gidley, 1926 en Gillette \& Ray, 1981- pág. 182, fig.86.

- Glyptotherium arizonae en Webb, 2001b, pág. 178, fig. 10.4A.

- Glyptotherium arizonae Gidley, 1926 en Cisneros, 2005 - pág. 243, fig. 4A, B, G-J

- Glyptotherium cf. G. arizonae Gidley, 1926 en Laurito et al., 2005, págs. 86-89, figs. 2-10.

- Glyptotherium cf. G. arizonae Gidley, 1926 en Valerio \& Laurito 2011a, págs. 134-137, figs. 2 (A y B).

Material: éste consta de cuatro osteodermos interiores y un osteodermo de posición incierta, depositados en la colección de fósiles de la Escuela Centroamericana de Geología de la Universidad de Costa Rica, bajo los números CF-5780, CF-5781, CF-5782, CF-5783, osteodermos interiores y el CF-5784 un osteodermo de posición incierta.

\section{Descripción}

En general las cuatro placas de osteodermos interiores presentan características similares, pero es la muestra número CF-5780 la que pre- 


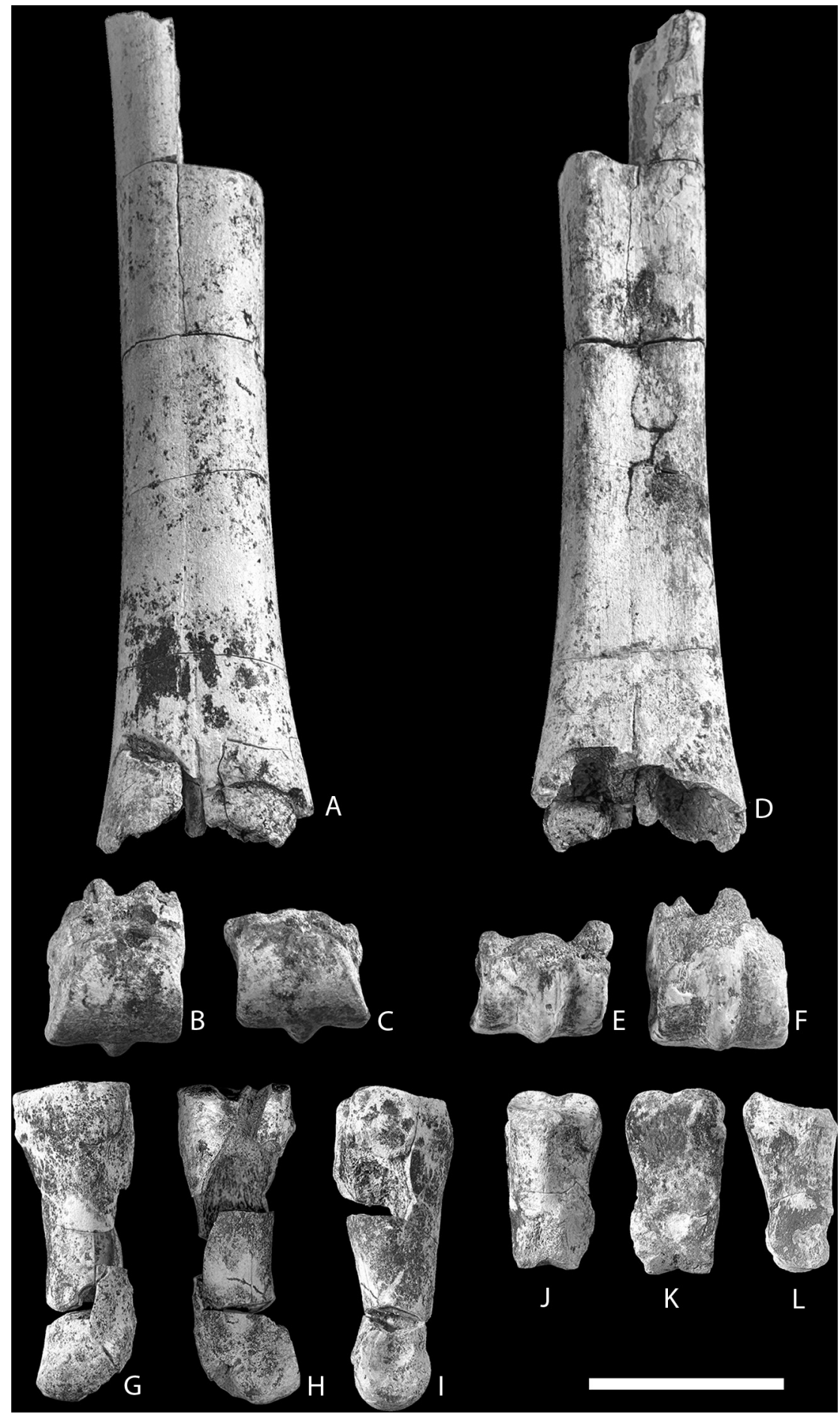

Fig. 3: Palaeolama mirifica (Simpson, 1929).: CF-5777 = metatarso fragmentado en vista anterior: A. metacarpo, B. cóndilo III, C. cóndilo IV; metatarso en vista plantar: D-F; CF-5778 = III falange proximal del pie en vistas: G. anterior, H. plantar, I. lateral; CF-5779 = III falange media podial en vistas: J. anterior, K. plantar, L. lateral. Escala visual: $4 \mathrm{~cm}$. 
senta mayor nitidez para la descripción, ya que las muestras CF-5781, CF-5782 y CF-5783 están cubiertas en la cara superior de una pátina de alteración por exposición a la meteorización.

Osteodermo CF-5780: constituido por 6 lados y de aproximadamente $47,51 \mathrm{~mm}$ de diámetro con un grosor de $16,24 \mathrm{~mm}$; la cara superior presenta una superficie plana, rugosa y punteada con un notable canal anular y 6 canales periféricos; la figura central es subcircular y en general más grande que las figuras periféricas; cada figura periférica consta de 5 lados y están un poco más elevadas que la figura central, las figuras periféricas son simétricas bilateralmente, en este ejemplar se observan 3 folículos pilosos, 2 sobre el canal anular y uno en la intersección de un canal periférico con el anular, la superficie inferior es punteada, débilmente cóncava y estriada (fig. 4A) .

Osteodermo CF-5781: mide aproximadamente $46,10 \mathrm{~mm}$ de diámetro, con un grosor de $15,47 \mathrm{~mm}$, debido a la pátina de alteración que cubre la cara exterior, solo se observa un folículo piloso, pero en general presenta todas las características descritas para la placa anterior (fig. 4B).

Osteodermo CF-5782: está constituido por 6 lados y un diámetro aproximado de $47,14 \mathrm{~mm}$, con un grosor de $15,07 \mathrm{~mm}$, solo se observa un folículo piloso ya que deben estar cubiertos por la pátina de alteración, pero en general se observan muchas de las características vistas en el ejemplar CF-5780 (fig. 4C).

Osteodermo CF-5783: constituido por 6 lados y un diámetro aproximado de 51,43 mm, con un grosor de $15,81 \mathrm{~mm}$, es la placa más grande $\mathrm{y}$ la menos visible debido a la pátina de alteración que la cubre (fig. 4D).

Osteodermo CF-5784: de posición incierta, elongado con un largo de $41,17 \mathrm{~mm}$, este es romo en uno de sus extremos, con un ancho de $32,12 \mathrm{~mm}$ y un grosor de $18,85 \mathrm{~mm}$, la cara superior presenta grandes folículos pilosos en dirección de la elongación, la superficie inferior presenta una estructura más compacta y lisa con pequeños forámenes vasculares y un ancho de 23,50 mm (figs. 3E-F).

\section{Discusión}

Las placas recolectadas son de buen grosor, tamaño y presentan 6 lados, en ellas sobresale su figura central plana, más grande y levemente más baja que las 6 figuras periféricas, estas últimas son simétricas bilateralmente, caracterizándose por su notable canal anular y canales periféricos propios de la especie Glyptoterium arizonae (Gidley, 1926) sensu Gillette \& Ray (1981).

La especie $G$. arizonae se diferencia de Glyptoterium texanun (Osborn, 1903) Gillette \& Ray (1981), en que las placas de G. texanum son más pequeñas y presentan figuras centrales más grandes que las figuras periféricas; aparte de ser convexas y sobresalir en elevación; además su canal anular y radial son poco profundos y en algunos casos mal definidos. En cuanto a Glyptoterium floridanum (Simpson, 1929) la diferencia radica en que presenta osteodermos más pequeños con figuras centrales ligeramente cóncavas, de similar tamaño y un poco más elevadas que las figuras periféricas.

Respecto a G. mexicanum y G. cylindricum sus escudos son muy similares a G. arizonae, pero se descartan por ser formas de edad Rancholabreano, es decir mucho más recientes.

Las placas de Puente de Piedra de Grecia, están muy relacionadas con la edad del hallazgo de Glyptoterium cf. G. arizonae Gidley, 1926 y Pachyarmatherium leiseyi Downig \& White (1995) de Buenos Aires de Palmares y descritas por Laurito et al. (2005) y Valerio \& Laurito (2011a).

\section{Distribución paleogeográfica}

Se acepta que el género Glyptotherium Osborn, 1903, evolucionó en América del Norte a partir de un ancestro suramericano y luego migró de manera inversa hacia América del Sur hace 2,7 M.a. sensu Carlini et al., 2008. Este género se ha registrado en el Blancano Tardío e Irvingtoniano Temprano de la Florida (Webb, 1974a; Gillette \& Ray, 1981), en el Irvingtoniano Temprano de Arizona y Oklahoma (Gillette \& Ray, 1981); así como en el Blancano 


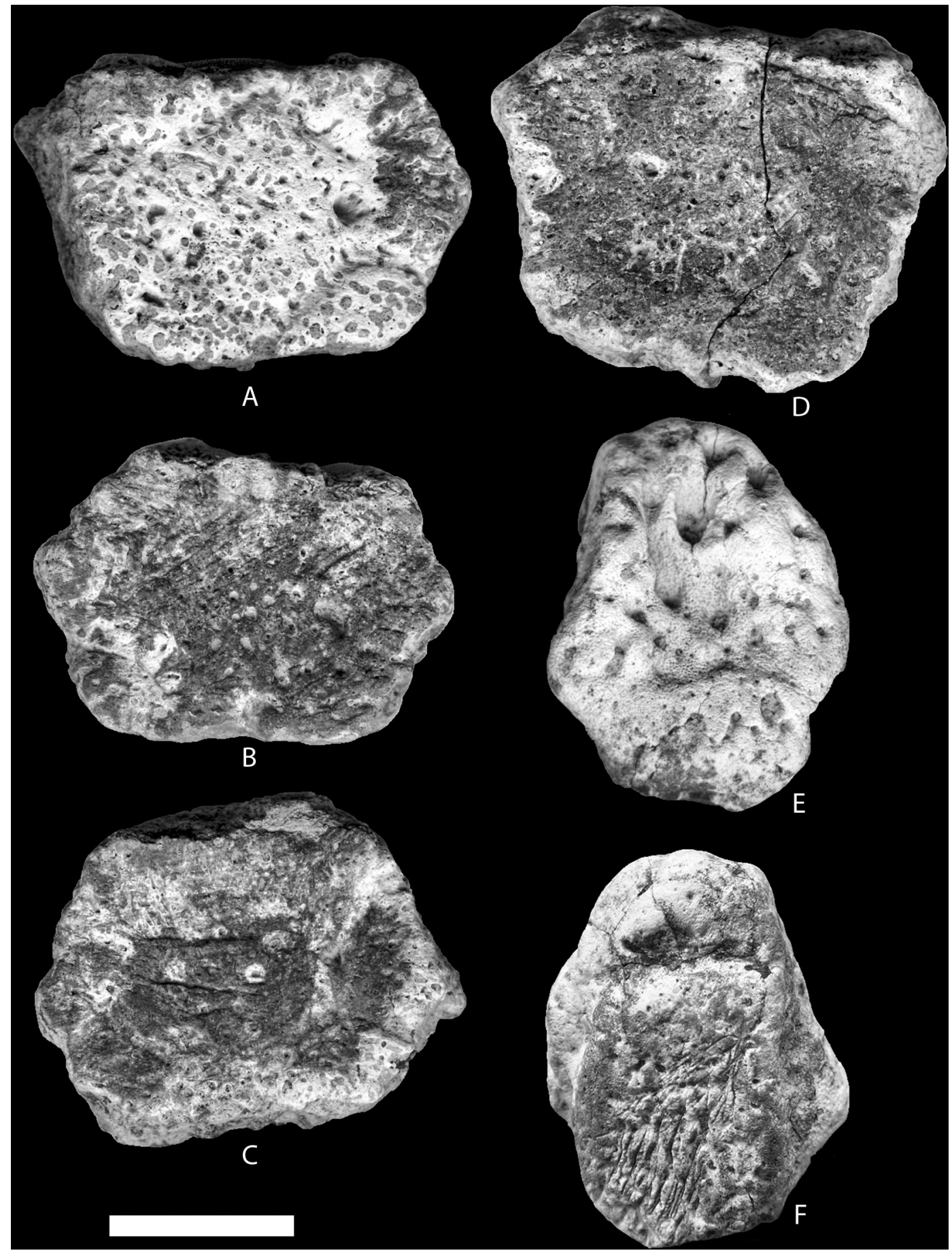

Fig. 4: Osteodermos de Glyptotherium aff. G. arizonae: A. CF-5780; B, CF-5781; C, CF-5782 y D, CF-5783, todos en vista dorsal; CF-5784, osteodermo de posición incierta en vistas: E. dorsal y F. ventral. Escala visual $2 \mathrm{~cm}$. 
Tardío e Irvingtoniano Temprano de Nuevo México (Morgan \& Lucas, 2003).

El registro más antiguo del género Glyptotherium, procede de la localidad de Rancho El Ocote de edad Blancano Temprano en el Centro de México, alrededor de los 3,1 y 3,9 M.a. sensu Flynn et al., 2005; también se ubica en el Pleistoceno Tardío (Rancholabreano) de Puebla, Sonora e Hidalgo (Tovar et al., 2007; Mead et al., 2007; Bravo-Cuevas et al., 2009).

En América Central el género Glyptotherium se ha registrado en el Pleistoceno Tardío de Guatemala cerca de la localidad de Santa Amelia (Woodburne, 1969) y en Chivacabé, Huehuetenango (Mead et al., 2012); Pleistoceno Tardío de Honduras en las localidades de Yecoronte y La Majadita, y en El Salvador en la localidad de Hormiguero (McGrew, 1942; Webb \& Perrigo, 1984; Jackson \& Fernández, 2005); en el Blancano Tardío de El Salvador (Cisneros, 2005); en Costa Rica se ubica en el Pleistoceno de la localidad de Chachagua (Valerio et al., 2005), en la localidad fosilífera del río Nacaome en la Península de Nicoya (Valerio \& Laurito, 2011b) y Pleistoceno Temprano de Buenos Aires de Palmares (Laurito et al., 2005; Valerio \& Laurito, 2011a); en Panamá se registra en el Pleistoceno de El Hatillo (Gazin, 1956).

La especie Glyptotherium arizonae Gidley, 1926 se presenta en el Blancano Tardío e Irvingtoniano Temprano de La Florida (Webb, 1974a) y Nuevo México (Morgan \& Lucas, 2003); Irvingtoniano de Arizona y Oklahoma (Gillette \& Ray, 1981), en los Estados Unidos.

En América Central Glyptotherium arizonae Gidley, 1926, se registra en el Pleistoceno Temprano-Medio de la localidad fosilífera del Río Tomayate en El Salvador (Cisneros, 2005), el Blancano Tardío - Irvingtoniano Temprano de la localidad de Buenos Aires de Palmares (Laurito et al., 2005; Valerio \& Laurito, 2011a) y la localidad de Puente Piedra de Grecia en el presente trabajo.

\section{CONCLUSIONES}

Los presentes hallazgos constituyen el segundo registro fósil de Camelidae Lamini y el primero de la especie Palaeolama mirifica (Simpson, 1929) para Costa Rica que junto a Glyptodontidae,
Glyptoterium aff. arizonae Gidley, 1926, son indicativos de la megafauna que habitó el territorio costarricense durante el Pleistoceno.

La localidad de Puente de Piedra de Grecia, sitio del hallazgo de estas dos muestras, está relacionada geológicamente con la localidad de Buenos Aires de Palmares, asociadas a depósitos lacustres, lugar en donde se hallaron osteodermos de Pachyarmatherium leiseyi Downing \& White, 1995 y Glyptotherium cf. arizonae Gidley, 1926; con base en esta asociación le fue asignada una "edad mamífero norteamericana" Blancano Tardío o Irvigtoniano Temprano sensu Laurito et al. (2005) y confirmada por Valerio \& Laurito (2011a).

Con base en lo anterior se asume que la edad del hallazgo de Palaeolama mirifica (Simpson, 1929) y Glyptoterium aff. arizonae Gidley, 1926, debe estar relacionada a la edad mamífero del lacustre de Palmares, Irvingtoniano Temprano.

\section{AGRADECIMIENTOS}

Al grupo de trabajo de mapeo geológico de la hoja Naranjo coordinado por la geóloga Ana Sofía Huapaya Rodríguez-Parra, quienes recolectaron las muestras. Al compañero geólogo Germán González M. por su ayuda en el trabajo de campo. Al paleontólogo César Laurito Mora por el montaje de las láminas. A Spencer Lucas por sus acertadas sugerencias.

\section{REFERENCIAS}

BRAVO-CUEVAS, V.M., ORTIZ-CABALLERO, E., \& CABRAL-PERDOMO, M. A., 2009: Glyptodontes (Xenarthra, Glyptodontidae) del Pleistoceno Tardío (Rancholabreano) de Hidalgo, Centro de México.- Bol. Soc. Geol. Mexicana, 61(2): 267-276.

CARLINI, A.A., ZURITA, A.E. \& AGUILERA, O.A., 2008: North American Glyptodontines (Xenarthra, Mammalia) in the upper Pleistocene of northern South America.- Paläontologische Zeitschrift, 82(2): 125-138. 
CISNEROS, J.C., 2005: New Pleistocene Vertebrate Fauna from El Salvador.- Rev. Bras. Paleontol. 8(3): 239-255.

FLYNN, J.J., KOWALLIS, B.J., NUÑEZ, C., CARRANZA-CASTAÑEDA, O., MILLER, W.E., SWISHER III, C.C. \& LINDSAY, E., 2005: Geochronology of Hemphillian-Blancan age strata, Guanajuato, Mexico, and implications for timing of the Great American Biotic Interchange.- J. Geol. 113: 287-307.

GAZIN, C.L., 1956: Exploration for the remains of giant ground sloths in Panama.Smithsonian Inst. Annual Report, 4272: 341-354.

GIDLEY, J.W., 1926: Fossil Proboscidea and Edentata of the San Pedro Valley, Arizona.USGS Professional Paper, 140B: 83-95.

GILLETTE, D.D. \& RAY C.E., 1981: Glyptodonts of North America.- Smithsonian Contributions to Paleobiol. 40: 1-255.

HIBBARD, C.W. \& DALQUEST, W.W., 1962: Artiodactyls from the Seymour Formation of Knox County, Texas.- Papers of the Michigan Academy of Sci. Arts and Letters, 47: 83-99.

HULBERT, R.C. \& PRATT, A.E., 1998: New Pleistocene (Rancholabrean) vertebrate faunas from coastal Georgia.- J. Vertebrate Paleontol. 18(2): 412-429.

JACKSON, D.R. \& FERNANDEZ, E., 2005: A small Pleistocene mammalian megafauna from southern Honduras.- Bull. Florida Mus. Nat. Hist. 45(4): 267-276.
LAURITO, C.A. 1993: Análisis topológico y sistemático del toxodonte de Bajo de los Barrantes, provincia de Alajuela, Costa Rica.- Rev. Geol. Amér. Central, 16: 61-68.

LAURITO, C.A., VALERIO, A. L. \& PÉREZ, E. A., 2005: Los xenarthras fósiles de la Localidad de Buenos Aires de Palmares (Blancano Tardío - Irvingtoniano Temprano), Provincia de Alajuela, Costa Rica.- Rev. Geol. Amér. Central, 33: 83-90.

LAURITO, C.A. \& VALERIO, A. L., 2010: Los caballos fósiles de la Formación Curré, cantón de Coto Brus, Costa Rica.- 131 págs. Mus. Nac. Costa Rica, San José.

LAURITO, C.A. \& VALERIO, A. L., 2012: Paleobiogeografía del arribo de mamíferos suramericanos al sur de América Central de previo al Gran Intercambio Biótico Americano: un vistazo al GABI en América Central.- Rev. Geol. Amér. Central, 46: 123-144.

MACGREW, P.O., 1942: Field Museum paleontological expedition to Honduras.- Science, New Series, 96: 85.

MEAD, J.I., BAEZ, A., SWIFT, S. L., LOHSE, J., \& PAIZ, L., 2012: Late Pleistocene mammals from Chivacabé, Huehuetenango, Guatemala.- Rev. Mexicana Cien. Geol. 29(2): 319-329.

MEAD, J.I., SWIFT, S.L., WHITE, R. S., MCDONALD, H.G. \& BAEZ, A., 2007: Late Pleistocene (Rancholabrean) Glyptodont and Pampathere (Xenarthra, Cingulata) from Sonora, México.- Rev. Mexicana Cien. Geol. 24: 439-449. 
MORGAN, G.S. \& LUCAS S.G. 2003: Mammalian biochronology of Blancan and Irvingtonian (Pliocene and early Pleistocene) faunas from New Mexico.Bull. Amer. Mus. Nat. Hist. 279: 269-320.

ROTH, J.A.\& LAERM, J., 1980: A late Pleistocene vertebrate Assemblage from Edisto Island, South Carolina.- Brimleyana, 3: 1-29.

RUEZ, D.R., JR., 2001: Early Irvingtonian (latest Pliocene) rodents from Inglis $1 \mathrm{C}$, Citrus County, Florida.- J. Vertebrate Paleontol. 21(1): 153-171.

RUEZ, D.R., JR., 2002: Mammalian taphonomy of the early Irvingtonian (late Pliocene) Inglis 1C fauna (Citrus County, Florida).Southeastern Geol. 41(3): 159-168.

RUEZ, D.R., JR., 2005: Earliest record of Palaeolama (Mammalia, Camelidae) with comments on "Palaeolama" guanajuatensis.- J. Vertebrate Paleont. 25(3): 741-744.

SIMPSON, G.G., 1929: Pleistocene mammalian fauna of the Seminole Field, Pinellas County, Florida.- Amer. Mus. Nat. Hist. Bull. 56: 561-599.

SLAUGHTER, B. H., 1966: The Moore Pit Local fauna; Pleistocene of Texas.- J. Paleontol. 40(1): 78-91.

TOVAR, R. E., MONTELLANOBALLESTEROS M. \& CORONA-M, E., 2007: Fauna Pleistocénica de Santa Cruz Nuevo, Puebla, México. En E. DÍAZMARTÍNEZ \& RÁBANO, I. (eds): $4^{\text {th }}$ European Meeting on the Paleontology and Stratigraphy of Latin America.- Bol. Inst. Geol. y Minero de España, 393-397.
VALERIO, A.L. \& LAURITO, C.A., 2004: Revisión del primer hallazgo de Eremotherium laurillardi (Xenarthra: Megatheriidae) en Costa Rica. Provincia de Alajuela, Costa Rica.- Brenesia, 62: 83-88.

VALERIO, A.L. \& LAURITO, C.A., 2011a: Nuevos hallazgos de Mammalia, Xenarthra (Cingulata) y confirmación del Registro de Pachyarmatherium leiseyi Dosning \& White, 1995 en la localidad de Buenos Aires de Palmares, Provincia de Alajuela, Costa Rica.- Rev. Geo. Amér. Central, 44: 131-139.

VALERIO, A.L. \& LAURITO, C.A., 2011b: El registro fósil de Glyptotherium floridanum (Xenarthra, Cingulata, Glyptodontidae) en el Cuaternario de Costa Rica, América Central.- Rev. Geol. Amér. Central, 45: 141-145.

VALERIO, A.L., LAURITO, C.A. \& GÓMEZ, L.D., 2005: Un Gliptodonte (Xenarthra, Cingulata) de la Localidad de Chachagua, Provincia de Alajuela, Costa Rica.- Rev. Geo. Amér. Central, 32: 61-63.

WEBB, S.D., 1974a: Chronology of Florida Pleistocene Mammals.- En WEBB, S.D. (ed.): Pleistocene Mammals of Florida.- The University of Florida Press, Gainesville: 5-31.

WEBB, S.D., 1974b: Pleistocene Llamas of Florida, with a brief review of the Lamini.En WEBB, S.D. (ed.): Pleistocene Mammals of Florida.- The University of Florida Press, Gainesville: 170-213.

WEBB, S.D., 2001a: Artiodactyla.- En: HULBERT, R.C. (ed.): The fossil verte- 
brates of Florida.- Univ. Press of Florida, Gainesville, 242-279.

WEBB, S.D., 2001b: Xenarthra.- En: HULBERT, R.C. (ed.): The fossil vertebrates of Florida.- Univ. Press of Florida, Gainesville, 175-187.

WEBB, D.S. \& PERRIGO, S. 1984: Late Cenozoic vertebrates from Honduras and El Salvador.J. Vertebrate Paleontol. 4(2): 237-254.
WEBB, D.S. \& STEHLI, F.G. 1995: Selenodont artiodactyla (Camelidae and Cervidae) from the Leisey Shell Pits, Hillsborough County, Florida.Bull. Florida Mus. Nat. Hist. 37(2): 621-643.

WOODBURNE, M. O., 1969: A late Pleistocene Occurrence of the Collared Peccary, Dicotyles tajacu, in Guatemala.- J. Mammalogy, 50(1): 121-25. 